\title{
Joint Guidance on Peptide Receptor Radionuclide Therapy in Neuroendocrine Tumors
}

A

practical guidance document on peptide receptor radionuclide therapy (PRRNT) in neuroendocrine tumors is being published this month in the European Journal of Nuclear Medicine and Molecular Imaging (1). This document is the result of close collaboration among experts in the field of PRRNT from the International Atomic Energy Agency; the Radionuclide Therapy, Oncology, Dosimetry, and Radiopharmacy Committees of the European Association of Nuclear Medicine; and the Society of Nuclear Medicine and Molecular Imaging.

This is a guidance document and not an organizational guideline. As such, it did not go through the formal process required for guidelines. In addition, the radiopharmaceuticals used for the diagnostic and therapeutic procedures addressed in this guidance document are not approved by the Food and Drug Administration (FDA) in the United States. Therefore, in the United States these procedures can be performed only by physicians enrolled in an investigational protocol pursuant to a valid Investigational New Drug application or Radioactive Drug Research Committee approval and under the purview of an appropriate Institutional Review Board.

The abstract is a good summary of the content of the guidance:

...PRRNT. . is a molecularly targeted radiation therapy involving the systemic administration of a radiolabelled peptide designed to target with high affinity and specificity receptors overexpressed on tumours. PRRNT employing the radiotagged somatostatin receptor agonists ${ }^{90}$ Y-DOTATOC $\left(\left[{ }^{90} \mathrm{Y}_{-D O T A}\right) \mathrm{Tyr}_{3}\right]$-octreotide) or ${ }^{177} \mathrm{Lu}$-DOTATATE $\left(\left[{ }^{177} \mathrm{Lu}-\mathrm{DOTA}{ }_{0}, \mathrm{Tyr}_{3}, \mathrm{Thr}_{8}\right]\right.$-octreotide or $\left[{ }^{177} \mathrm{Lu}\right.$-DOTA $\left.0, \mathrm{Tyr}_{3}\right]$-octreotate) have been successfully used for the past 15 years to target metastatic or inoperable neuroendocrine tumours expressing the somatostatin receptor subtype 2. Accumulated evidence from clinical experience indicates that these tumours can be subjected to a high absorbed dose which leads to partial or complete objective responses in up to $30 \%$ of treated patients. Survival analyses indicate that patients presenting with high tumour receptor expression at study entry and receiving ${ }^{177} \mathrm{Lu}$-DOTATATE or ${ }^{90}$ Y-DOTATOC treatment show significantly higher objective responses, leading to longer survival and improved quality of life. Side effects of PRRNT are typically seen in the kidneys and bone marrow. These, however, are usually mild provided adequate protective measures are undertaken. Despite the large body of evidence regarding efficacy and clinical safety, PRRNT is still considered an investigational treatment and its implementation must comply with national legislation, and ethical guidelines concerning human therapeutic investigations. This guidance was

Received Mar. 14, 2013; accepted Mar. 14, 2013.

For correspondence or reprints contact: Dominique Delbeke, Department of Radiology, Vanderbilt University Medical Center, 21st Ave. S. and Garland, Nashville, TN 37232.

E-mail: dominique.delbeke@vanderbilt.edu

Published online Mar. 27, 2013.

COPYRIGHT $\odot 2013$ by the Society of Nuclear Medicine and Molecular Imaging, Inc.

DOI: 10.2967/jnumed.113.123190 formulated based on recent literature and leading experts' opinions. It covers the rationale, indications and contraindications for PRRNT, assessment of treatment response and patient follow-up. This document is aimed at guiding nuclear medicine specialists in selecting likely candidates to receive PRRNT and to deliver the treatment in a safe and effective manner. This document is largely based on the book published through a joint international effort under the auspices of the Nuclear Medicine Section of the International Atomic Energy Agency.

Although PRRNT is not approved in many countries, including the United States, it is feasible to refer patients to sites in Europe where they can receive this therapy. It is essential that the patients have been evaluated with ${ }^{111} \mathrm{In}$ pentetreotide (OctreoScan; Mallinckrodt Inc.) or ${ }^{68} \mathrm{Ga}$-DOTA somatostatin receptor scintigraphy and have biopsy-proven neuroendocrine tumor with high uptake, usually grade 3 or 4 , before referral. The ${ }^{68} \mathrm{Ga}$-DOTA somatostatin receptor imaging radiopharmaceuticals are still investigational in the United States as well. ${ }^{111} \mathrm{In}$-pentetreotide is the only FDA-approved somatostatin receptor radiopharmaceutical.

Clinical trials with these agents are getting under way in the United States. The ${ }^{177}$ Lu-DOTATATE study is an international phase 3 multicenter trial for patients with midgut neuroendocrine tumors and metastases; patients are randomized to ${ }^{177} \mathrm{Lu}$-DOTATATE in 4 doses administered every $4 \mathrm{wk}$ or $60 \mathrm{mg}$ of octreotide acetate (Sandostatin LAR; Novartis Pharmaceuticals Corp.) every 4 wk, with time to progression and overall survival as endpoints. This trial is expected to open in 13 centers in the United States and should provide sufficient safety and efficacy data to lead to FDA approval. The ${ }^{90}$ Y-DOTATOC study is an open-label phase 2 study expected to open in September 2013.

PRRNT is a powerful concept that will become increasingly important as new agents continue to be developed and approved. Similar to ${ }^{131}$ I therapy for hyperthyroidism and thyroid cancer, it provides a magic bullet that treats the tumor without significantly affecting normal tissues.

\section{REFERENCE}

1. Zaknun JJ, Bodei L, Mueller-Brand J, et al. The joint IAEA, EANM, and SNMMI practical guidance on peptide receptor radionuclide therapy (PRRNT) in neuroendocrine tumours. Eur J Nucl Med Mol Imaging. February 7, 2013 [Epub ahead of print].

Dominique Delbeke Vanderbilt University Medical Center Nashville, Tennessee

Michael M. Graham University of Iowa Iowa City, Iowa 